Nouvelles perspectives en sciences sociales

Revue internationale de systémique complexe et d'études relationnelles

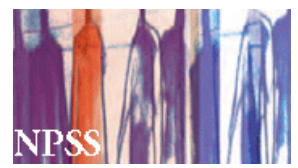

\title{
La notion de modèle en philosophie des sciences
}

\section{Jean Leroux}

Volume 7, numéro 2, mai 2012

Sur le thème de la modélisation

URI : https://id.erudit.org/iderudit/1013054ar

DOI : https://doi.org/10.7202/1013054ar

Aller au sommaire du numéro

\section{Éditeur(s)}

Prise de parole

\section{ISSN}

1712-8307 (imprimé)

1918-7475 (numérique)

Découvrir la revue

\section{Citer cet article}

Leroux, J. (2012). La notion de modèle en philosophie des sciences. Nouvelles perspectives en sciences sociales, 7(2), 49-65. https://doi.org/10.7202/1013054ar

\section{Résumé de l'article}

Nous voulons faire valoir le caractère central de la notion de modèle en philosophie des sciences. Nous aborderons d'abord la question du rapport qu'entretiennent les notions de modèle et de théorie, pour ensuite distinguer dans l'utilisation qu'en a fait la philosophie des sciences, l'acception du terme en tant que conception générale de celle qui prévaut maintenant dans les approches contemporaines. Nous présentons de façon critique et informelle les différentes voies que l'analyse épistémologique a adoptées afin de caractériser les modèles associés aux théories scientifiques, et nous en discutons les enjeux. Enfin, et à titre d'exemple, nous exposons comment le débat sur la question du réalisme scientifique s'articule sur la notion de modèle. 


\title{
La notion de modèle en philosophie des sciences
}

\author{
JeAn LeRoux \\ Université d'Ottawa
}

\section{Introduction}

Tous voulons apporter des considérations d'ordre général sur le rôle de la notion de modèle en philosophie des sciences. Cette question est distincte de celle du rôle de la notion de modèle en science. La science connaît une pluralité d'acceptions du terme "modèle ", qui peut renvoyer tout autant à de purs symbolismes qu'à des entités conceptuelles ou concrètes. Cette polysémie est évidemment liée à la diversité des fonctions assignées aux modèles scientifiques, dont la plus importante pour notre propos est la fonction heuristique. L'ébauche de modèles guide en effet la recherche des théories émergentes, tout comme elle certifie l'interprétation et l'applicabilité des théories acceptées; on parle ainsi de modèles théoriques et de modèles empiriques de ces théories. Une telle façon de s'exprimer suppose cependant une distinction ou un écart entre la théorie scientifique et ses modèles, distinction qui est précisément mise en cause par la conception aujourd'hui généralement adoptée des théories scientifiques, la conception "sémantique "; dans cette approche, les théories scientifiques sont directement identifiées 
à leurs modèles, et non à leurs formulations linguistiques. C'est dire que ce qui est ici directement visé est la notion même de théorie.

La philosophie des sciences en tant qu'entreprise métathéorique doit, tout comme la science, se fixer sur un cadre d'analyse lui permettant d'identifier et de singulariser son objet d'étude. Sur le plan historique, divers développements ont mené le mouvement qui a donné à la philosophie des sciences ses impulsions majeures au début du $\mathrm{XX}^{\mathrm{e}}$ siècle, l'empirisme logique, à adopter une approche des théories scientifiques en tant qu'entités linguistiques ou langagières, "langage " étant ici conçu largement en tant qu'ensemble de signes ou système symbolique. L'essor de la logique formelle et, surtout, ce qu'il a été convenu d'appeler le "tournant linguistique " de la philosophie ont sans doute été les facteurs les plus déterminants dans l'orientation de la philosophie des sciences sur la voie qu'avaient auparavant emprunté la philosophie des mathématiques et la tradition naissante de l'investigation des systèmes axiomatiques formels. Il devint usuel de considérer les théories scientifiques - les théories visées étant de prime abord celles de la physique mathématique - en tant que systèmes axiomatiques formels. Cette approche des théories scientifiques reçut plusieurs appellations, dont la plus usitée est celle de modèle empiriste standard des théories scientifiques ${ }^{1}$.

Parmi les diverses appellations du modèle empiriste logique des théories scientifiques, mentionnons : conception reçue et conception syntaxique ou énonciative ["statement view"]. Les formulations élaborées par Rudolf Carnap, "The Methodological Character of Theoretical Concepts ", dans Herbert Feigl et Michael Scriven (dir.), The Foundations of Science and the Concepts of Psychology and Psychoanalysis, Minneapolis, University of Minnesota Press, coll. "Minnesota Studies in the Philosophy of Science ", vol. 1, 1956, p. 38-76, et par Carl Hempel, "The Theoretician's Dilemma. A Study in the Logic of Theory Construction ", dans Herbert Feigl, Michael Scriven et Grover Maxwell (dir.), Concepts, Theories, and the Mind-Body Problem, Minneapolis, University of Minnesota Press, coll. "Minnesota Studies in the Philosophy of Science ", vol. 2, 1958, p. 37-98, font figure de version finale, dans la mesure où le modèle fut généralement délaissé dans les années suivantes. 


\section{Modèles en tant que conceptions générales}

C'est donc à l'occasion du concept même de théorie que nous rencontrons le terme modèle en philosophie des sciences. Le modèle empiriste logique d'un langage à double palier observationnel et théorique des théories scientifiques eut, pour ainsi dire, un compagnon d'infortune, le modèle logique d'explication scientifique; ce dernier, élaboré par Hempel, posait une identité de structure logique entre la prédiction des faits et leur explication $^{2}$.

Ces deux modèles épistémologiques ont été largement diffusés, mais la notion de modèle ici mise en cause n'est pas celle qui nous intéresse à proprement parler. Dans les expressions « modèle empiriste standard des théories scientifiques » et " modèle déductif-nomologique d'explication scientifique ", on peut tout simplement remplacer le terme "modèle " par " conception générale ». Le premier terme était utilisé plutôt que le second en raison du fait que cette conception se voulait précise et qu'elle formulait des conditions que devaient satisfaire toute théorie et toute explication scientifiques. De ces modèles, dont les insuffisances ont été amplement discutées, nous voulons simplement souligner le caractère canonique. Ils ne prétendaient pas décrire les théories et les explications scientifiques telles qu'on les retrouve effectivement formulées en science; il s'agissait de reconstructions logiques, l'objectif étant que l'on pouvait, pour fins d'analyse épistémologique, redonner les théories et explications scientifiques sous cette forme. En fait, la conception élaborée par l'empirisme logique de ce qu'est une théorie scientifique fut à l'origine calquée sur la théorie de la relativité qui, on le sait,

$\overline{2}$ On pourrait ajouter au modèle logique d'explication des faits les modèles classiques de réduction inter-théorique proposés par Ernest Nagel, The Structure of Science: Problems in the Logic of Scientific Explanation, New York, Hartcourt, Brace \& World, 1961, et perpétués par Wesley Salmon, Causality and Explanation, Oxford, Oxford University Press, 1998, ceux-ci étant essentiellement des généralisations, des faits aux théories, du concept logique d'explication formulé par Carl Hempel, "Studies in the Logic of Explanation », Philosophy of Science, vol. 15, 1948, p. 135-75; Carl Hempel, Aspects of Scientific Explanation and Other Logical Essays, New York, Free Press, 1965. 
comportait certaines contraintes formelles pour toute théorie physique. Quant au modèle d'explication proposé par Hempel, il visait à réhabiliter l'idée d'explication scientifique, laquelle avait été généralement délaissée au début du XX $\mathrm{X}^{e}$ siècle au profit d'une conception voulant que la tâche des sciences naturelles soit uniquement de décrire les phénomènes naturels, et non d'en rechercher les causes ou de les expliquer. L'infortune de ces approches empiristes logiques fut essentiellement due à leur incapacité d'intégrer des composantes d'ordre pragmatique, de sorte qu'en définitive, elles ne parvenaient pas à établir la distinction entre, d'une part, théorie formelle et théorie empirique, et, d'autre part, explication et description structurelle.

\section{Modèles au sens de la théorie des modèles}

Le modèle empiriste logique des théories scientifiques n'est donc pas un modèle au sens strict que nous voulons présenter. Cette conception transpose en fait du domaine formel (logique et mathématique) au domaine empirique (scientifique) la notion de théorie que la logique avait développée lors de l'essor de la problématique des fondements.

La notion de théorie en logique se définit concurremment à celle de langage formel. Un langage formel $L$ est un couple $<S, R f>$ où $S$ est un ensemble de signes (l'alphabet du langage formel) et $R_{f}$ est un ensemble de règles de formation (la syntaxe du langage) qui définissent récursivement la notion de formule bien formée. Un tel langage formel est donc déterminé en faisant entièrement abstraction de l'interprétation qu'on entend faire de ces signes. On peut doter une telle structure syntaxique d'une base logique en définissant un ensemble d'axiomes $A$ et un ensemble de règles d'inférence $R_{t f}$. conçues en tant que simples règles de transformation ou de réécriture : ces règles syntaxiques sont cependant choisies en fonction de leur interprétation visée, c'est-à-dire, des règles d'inférence déductive valides. Le couple $<A, R t f>$ forme une structure déductive qui détermine dans $L$ une classe d'énoncés close par la relation de déduction. Le qua- 
druplet $<S, R f, A, R t f>$ est une théorie axiomatique formelle. Dans le cas où $A$ est un ensemble d'axiomes logiques (dans le cas où $A=A_{l}$ ), cette structure correspond à une théorie logique. On peut concevoir une théorie scientifique mathématisée (une théorie physique, par exemple) comme un système axiomatique formel <S, Rf, $A l, A m, A p, R t f>$ où l'on a ajouté aux axiomes logiques $A_{l}$ un ensemble $A_{m}$ d'axiomes mathématiques et un ensemble $A_{p}$ d'axiomes physiques (les axiomes propres de la théorie).

La théorie ainsi logiquement reconstruite représente donc essentiellement un appareil déductif ou prédictif, qui est en même temps un appareil nomologique ou explicatif, selon les termes du modèle logique d'explication, et la question de son interprétation n'est traitée qu'une fois accomplie sa détermination syntaxique.

Bien que cette approche dicte que l'explicitation syntaxique (formalisation et axiomatisation) des théories scientifiques se fasse préalablement à leur analyse épistémologique, elle n'en procède pas moins à leur analyse sémantique. C'est dans ce cadre d'analyse - la sémantique logique ou "théorie des modèles " - qu’apparaît la notion de modèle qui est l'objet propre de notre propos. Nous voulons, dans ce qui suit, présenter informellement et à grands traits cette notion de modèle au sens de la sémantique logique, dans sa version ensembliste.

Si l'on convient que l'interprétation d'une théorie scientifique est donnée par les moyens que celle-ci utilise pour identifier ses référents, l'adoption d'une sémantique extensionnelle basée sur la notion de référence ou d'extension semble tout indiquée. L'analyse sémantique verra donc d'abord à établir un cadre pour traiter du domaine de référence d'une théorie scientifique.

La notion de départ est celle de structure. On convient aussi généralement que l'hypothèse scientifique porte sur la réalisation empirique de structures formelles mises en jeu par la théorie. L'hypothèse s'avère si les relations décrites par la théorie sont réalisées parmi les éléments du domaine visé d'explication. Une telle vision des choses s'accorde tout à fait au précepte structura- 
liste qui pose que tous les énoncés scientifiques sont des énoncés structuraux, c'est-à-dire qu'ils affirment que tel et tel domaine possède telle et telle structure, auquel cas ils sont vrais de ce domaine. Dans sa forme la plus simple, une structure, notée $<D, R i>$ est un ensemble $D$ d'objets (le domaine de la structure) muni d'un nombre $R_{i}$ de relations définies sur $D$. Dans un sens très large, une structure, c'est-à-dire une multitude d'objets où existent une variété de relations, représente un monde, un univers qui est à proprement parler un univers du discours.

Les structures deviennent des modèles lorsqu'on introduit l'élément sémantique (la notion de vérité). La théorie des modèles développée par la sémantique logique considère de telles structures comme domaines d'interprétation des langages formels. En assignant à chaque terme d'un langage formel sa référence et à chaque énoncé ses conditions de vérité, ce langage, qui n'était de prime abord qu'un simple système de signes non interprétés, un simple calcul, devient un langage interprété. Un modèle est une structure dont un énoncé ou ensemble d'énoncés (disons : les axiomes d'une théorie) est vrai; il constitue une réalisation de cet énoncé ou de cette théorie. Si on entend par contenu d'un énoncé l'état de choses qui existe lorsque cet énoncé est vrai, les modèles d'une théorie en représentent le contenu.

Cette notion de modèle, due à Tarski, permettait en logique d'établir la notion sémantique de consistance : une théorie est consistante si elle possède un modèle. Elle permettait aussi de définir la notion sémantique de conséquence logique : un énoncé $\beta$ est une conséquence logique d'un énoncé $\alpha$ si tous les modèles de $\alpha$ sont des modèles de $\beta$.

La philosophie empiriste des sciences a fait ample usage de cette approche dans l'analyse du contenu empirique des théories scientifiques ${ }^{3}$. À cet effet, on a eu recours à la notion de structures

3 Pour une exposition de la conception reçue dans le cadre de la théorie des modèles, voir Marian Przełęcki, The Logic of Empirical Theories, Londres, Routledge and Kegan Paul, 1969; Jean Leroux, Une Histoire comparée de la philosophie des sciences, vol. 2. L’Empirisme logique en débat, Québec, Presses de l'Université Laval, coll. «Logique de la science », 2010, chap. 1, en offre 
observationnelles, qui sont des structures composées uniquement d'objets observables et munies uniquement de relations observables. De telles structures observationnelles constituent le socle de la théorie scientifique, qui, évidemment, ne s'en tient pas à l'observable. La théorie enrichira ces structures d'objets et de relations inobservables pour dépeindre des modèles qui ne sont plus l'image manifeste du monde, mais son image scientifique; cette image scientifique est peuplée d'entités qui échappent à l'intuition et au sens commun et qui ne répondent qu'à des valeurs de rationalité. Les modèles observationnels ne sont qu'une partie, si infime soit-elle, des modèles entiers d'une théorie scientifique, dont on dit que le contenu "dépasse » l'expérience.

La notion de modèle joue donc un rôle essentiel dans la formulation exacte de la thèse empiriste logique, qui énonce que toute théorie scientifique peut être logiquement reconstruite en un formalisme qui possède des modèles observationnels; la reconstruction logique sert ainsi à établir la thèse de l'empirisme sémantique, à savoir que les assignations de référents et de valeur de vérité des termes et énoncés d'une théorie scientifique (donc: sa vérification) procèdent ultimement par voie empirique.

La notion de modèle ainsi conçue intervient également dans la formulation de desiderata épistémologiques centraux tels la consistance (au sens sémantique) et l'adéquation empirique : toute théorie scientifique doit posséder au moins un modèle, et les modèles observationnels de la théorie doivent " correspondre " aux structures concrètes observées ${ }^{4}$. La notion de modèle apparaît aussi dans la formulation de la thèse de la sous-détermination empirique des théories scientifiques : plusieurs modèles théoriques peuvent posséder les mêmes modèles observationnels.

\section{Modèles au sens structuraliste}

À partir des années 1970, on a généralement délaissé l'approche ci-dessus décrite au profit d'une conception des théories scienti-

une discussion succincte.

Sous ce terme " correspondre " se cachent tous les problèmes épistémologiques reliés à la volonté de maintenir en science la notion réaliste ou vérifactuelle de vérité (vérité en tant que correspondance au réel ou aux faits). 
fiques non plus en tant que structures d'énoncés, mais en tant que concepts structurels. La caractéristique individuante d'une théorie n'est plus la classe de ses affirmations (ses lois fondamentales ou ses axiomes), mais la classe de ses modèles. Si la mise au rancart du mouvement empiriste logique en philosophie des sciences fut accompagnée de l'abandon de la notion logique de théorie, elle n'a nullement signifié un renoncement à la notion de modèle; au contraire, cette dernière occupera dorénavant l'avant-scène d'un nouveau paradigme philosophique opérant sous le sigle de la conception sémantique des théories scientifiques.

Si l'on s'attarde un instant à la critique interne qu'eut à subir l'approche syntaxique, on peut convenir qu'il y avait erreur à considérer l'appareil nomologique (la théorie au sens du modèle logique) et l'appareil heuristique d'une théorie (son interprétation ou ses modèles au sens de la théorie des modèles) comme deux entités distinctes. La science explique et interprète le monde d'un seul mouvement, construction théorique et construction conceptuelle allant de pair. La science construit ses objets à même les théories dont on dit qu'elles portent sur ces objets, et un empirisme d'allégeance constructiviste verra une différence d'aspects, et non une différence de tâches, dans la distinction entre l'agencement théorique des énoncés et l'agencement heuristique des liens qu'ils ont avec l'expérience.

L'idée de base de la conception sémantique est qu'une théorie scientifique constitue une entité interprétée au départ, une structure conceptuelle abstraite, mathématiquement descriptible, qui est mise en relation avec un ensemble de phénomènes possibles, actuels ou visés. Cette façon de concevoir les théories scientifiques invite à se limiter aux modèles d'une théorie donnée sans avoir à en effectuer préalablement l'explicitation syntaxique formelle, comme le voulait la conception reçue. Diverses versions 
de cette approche furent élaborées aux États-Unis ${ }^{5}$, en Pologne ${ }^{6}$, en Allemagne ${ }^{7}$ et ailleurs; plusieurs autres se sont ajoutées pour maintenant constituer l'approche dominante en philosophie des sciences $^{8}$. Les différentes versions de cette approche ont en commun de suivre le précepte de Patrick Suppes enjoignant d'utiliser la mathématique (et non la métamathématique) comme instrument d'analyse; elles different par ailleurs selon le type

$5 \quad$ Voir Patrick Suppes, «A Comparison of the Meaning and Use of Models in Mathematics and the Empirical Sciences ", dans Hans Freudenthal (dir.), The Concept and the Role of Model in Mathematics and Natural and Social Science, Dordrecht, Reidel, 1961, p. 287-301; Patrick Suppes, "What is a Scientific Theory? ", dans Sidney Morgenbesser (dir.), Philosophy of Science Today, New York, Basic Books, 1967, p. 55-67; Bas van Fraassen, The Scientific Image, Oxford, Clarendon Press, 1980; Bas van Fraassen, "Empiricism in the Philosophy of Science ", dans Paul Churchland et Clifford A. Hooker (dir.), Images of Science: Essays on Realism and Empiricism with a Reply by Bas C. van Fraassen, Chicago, The University of Chicago Press, 1985, p. 245-308; Bas van Fraassen , "The Semantic Approach to Scientific Theories », dans Nancy J. Nersessian (dir.), The Process of Science, Dordrecht, Kluwer, 1987. p. 105124; Frederick Suppe, The Semantic Conception of Theories and Scientific Realism, Urbana, University of Illinois Press, 1989, et Ronald Giere, Explaining Science. A Cognitive Approach, Chicago, The University of Chicago Press. 1990.

6 Voir Marian Przełęcki. op. cit.

7 On a assisté dans les années 1970 à un véritable « mouvement structuraliste " en Europe. Initié par Joseph D. Sneed, The Logical Structure of Mathematical Physics, Dordrecht, Reidel, 1971, et Wolfgang Stemüller, The Structuralist View of Theories, Berlin, Springer, 1979. Ce mouvement a initialement repris à nouveaux frais plusieurs problématiques de l'empirisme logique (le problème des termes théoriques, la donnée du contenu empirique d'une théorie, la réduction inter-théorique); le mouvement culmine dans l'ouvrage de Wolfgang Balzer, Carlos Ulises Moulines et Joseph D. Sneed, An Architectonic for Science: the Structuralist Program, Dordrecht, Kluwer, 1967.

8 Patrick Suppes, Newton C. A. Da Costa et Steven French, "The Model Theoretic Approach in Philosophy of Science ", Philosophy of Science, vol. 57, 1990, p. 248-265; Steven French et James Ladyman, "Reinflating the Semantic Approach ", International Studies in the Philosophy of Science, vol. 13, 1999, p. 103-121; Newton C. A. Da Costa et Steven French, "Models, Theories, and Structures: Thirty Years on ", Philosophy of Science, vol. 67, Supplement. Proceedings of the 1998 Biennial PSA Meetings, Part II, 2000, p. 116-127, ainsi que Anjan Chakravartty, "The Semantic or ModelTheoretic View of Theories and Scientific Realism ", Synthese, vol. 127, 2001, p. 325-345, constituent d'excellentes études de synthèse, incluant les débats d'ordre technique concernant la différence entre les modèles au sens du structuralisme et les modèles au sens de la théorie des modèles. 
d'appareil mathématique qu'elles utilisent pour décrire directement ces modèles qui, maintenant, composent la théorie.

Sneed et le mouvement structuraliste européen ont d'abord décrit les modèles caractéristiques d'une théorie scientifique (la mécanique classique des particules) dans le cadre de la théorie naïve des ensembles. Suite à certains scrupules d'ordre logique, on a alors utilisé le cadre de la théorie typifiée formelle des ensembles (dans les lignes de Bourbaki), pour enfin adopter le cadre de la théorie des catégories qui offrait une présentation plus élégante. Cette version structuraliste ne réintégrait un élément linguistique qu'au niveau de la donnée de l'affirmation empirique d'une théorie scientifique. L'énoncé contingent d'une théorie empirique affirme que les structures observées appartiennent (au sens de la relation $\in$ de la théorie des ensembles) aux structures observationnelles qui peuvent être enrichies en modèles entiers de la théorie à l'aide de fonctions qui satisfont à un ensemble de contraintes formelles assurant (pour l'essentiel) le caractère contingent de l'énoncé.

Bas van Fraassen, qui fut certes, après Suppes, celui qui a le plus contribué à l'implantation de la conception sémantique, considère que la donnée des modèles d'une théorie scientifique se fait dans le même langage mathématique que la théorie utilise. La caractérisation d'une théorie physique comporte la donnée de trois éléments : un espace d'état $H$ (qui spécifie la sorte de systèmes auxquels la théorie s'applique), une ensemble $E$ d'énoncés élémentaires portant sur des grandeurs physiques mesurables, et une fonction de satisfaction $h$ qui représente le lien que la théorie établit entre ses modèles mathématiques et les résultats expérimentaux empiriques. Le triplet $\langle H, E, h>$ détermine un langage élémentaire dans lequel les affirmations empiriques de la théorie (et non la théorie entière) peuvent être formulées.

La question pertinente à notre propos est de savoir en quoi les modèles de l'approche sémantique diffèrent des modèles au sens de la théorie des modèles, lesquels sont déterminés en tant que domaines de validité des énoncés qui composent la théorie. Plusieurs de ces approches considèrent que la notion de modèle 
qu'elles utilisent équivaut à celle de la théorie des modèles (parfois appelée la notion modèle-théorétique de modèle); ils considèrent que les modèles mathématiquement descriptibles de la théorie scientifique sont ceux qu'une explication formelle du langage de celle-ci aurait dégagés, si on l'avait fait. De tels modèles ensemblistes se résument à des structures relationnelles telles que décrites ci-dessus.

On pourrait cependant opposer à toute conception qui n'intègre pas une dimension langagière aux théories scientifiques qu'elle ne mérite sa qualification d'approche sémantique qu'au sens contrefactuel du terme. Il s'agit à proprement parler d'une conception purement structuraliste où les modèles sont considérés non pas en tant qu'interprétations de la théorie, mais en tant qu'applications de la théorie qui servent à sa définition ${ }^{9}$. Si on écarte explicitement toute considération du langage d'une théorie, on peut difficilement prétendre procéder à une analyse sémantique de celle-ci. L'approche structuraliste s'épargne la grande question du lien entre langage et réalité (deux entités d'inégale nature), au profit espéré d'un cantonnement à la question du lien entre structures abstraites (théorie) et structures concrètes (réalité). Considérer la donnée de structures qui ne soient pas médiatisées par le langage incite à une forme de réalisme plus profond que le réalisme inhérent à l'utilisation de la théorie des modèles. Comment concevoir le lien entre les structures ou modèles observationnels de la théorie et les structures observées concrètes? L'approche structuraliste envisage la question en termes d'imbrication [embedment], de congruence ou d'appartenance : l'adéquation empirique d'une théorie au sens structuraliste signifie que les structures observées font partie des structures observationnelles qui représentent le contenu observable de la théorie. On passe de la correspondance au réel à l'inhérence au réel.

9 Voir Jean Leroux, "The Structuralist View of Theories and Empiricism ", Dialectica, vol. 41, 1987, p. 321-326, et Werner Diederich, "Realität und Modell. "Semantischer" und "strukturalistischer" Ansatz in der Wissenschaftstheorie ", Allgemeine Zeitschrift für Philosophie, vol. 14, 1989, p. 1-14. 
Comment des structures concrètes observées peuvent-elles faire partie d'une théorie, qui se compose de structures abstraites? Le renvoi à une faculté intellectuelle d'abstraction ne suffirait pas, car les modèles scientifiques sont aussi construits par voie d'idéalisations et d'analogies. Les questions centrales de l'approche structuraliste doivent reprendre à nouveaux frais des problématiques qui ont une lourde charge de questions traditionnelles non résolues.

La conception purement structuraliste peine présentement à apporter une réponse plausible à la question de savoir en quoi des structures (les théories) peuvent représenter des structures une réédition du problème de la représentation ${ }^{10}$. On notera également que le prédicat de vérité ne s'applique pas aux concepts, mais aux énoncés. Un concept s'applique avec plus ou moins de succès à un domaine donné. En ce sens, la conception des théories en tant que structures conceptuelles divorcées du langage est idoine au point vue instrumentaliste qui ne retient de la science que sa systématisation de l'expérience. Pourtant, à l'exception notoire de van Fraassen, les tenants de l'approche structuraliste défendent pour la plupart une forme ou l'autre de réalisme scientifique.

\section{Modèles et réalité}

La question du réalisme scientifique s'articule sur la façon de concevoir le rapport qui peut bien exister entre la théorie scientifique et la réalité, conçue en tant qu'entité extra-linguistique. Comment comprendre la relation qui existe entre les entités qui peuplent le monde ou la réalité, et celles qui peuplent nos théories, nos modèles de la réalité? À défaut de se demander, lorsqu'on adopte la conception langagière des théories, s'il existe une forme de réalité qui ne soit pas médiatisée par le langage, on peut se demander, lorsqu'on adopte la conception sémantique des théories, s'il existe une forme de réalité qui ne soit pas modélisée. Un

$10 \quad$ Le plus récent ouvrage de Bas van Fraassen, Scientific Representation: Paradoxes of Perspective, Oxford, Clarendon Press, 2008, porte expressément sur cette problématique. 
simple coup d'œil sur la pratique scientifique suffit à apporter une réponse négative à cette dernière question; les données de base $[$ data $]$ d'une théorie scientifique doivent être considérées comme d'emblée modélisées [model data]. C'est dire que la relation entre la théorie et son objet doit être étudiée sur le mode des relations inter-structurelles.

La relation de base est celle d'identité structurelle ou d'isomorphisme. Deux structures sont isomorphes si les relations qui prévalent dans le domaine de la première sont préservées par les relations tenant dans le domaine de la seconde, laquelle peut être constituée d'entités entièrement différentes. Du point de vue de la sémantique logique, qui introduit de pénétrantes réflexions sur la question, l'isomorphisme de deux modèles $M_{1}$ et $M_{2}$ est intéressante dans la mesure où tout ce qui est vrai de $M_{1}$ est vrai de $M_{2}$. Au cas où nous considérons que modèle et réalité sont isomorphes, tout ce qui est vrai du modèle est vrai de la réalité - ce qui serait le point de vue du réalisme scientifique intégral qui pose d'un même geste et la vérité de la théorie et l'existence effective des entités théoriques décrites par la théorie. C'est dire que les modèles entiers de la théorie, y compris toute leur part d'inobservable, sont effectivement réalisés dans le monde; ils constituent une réalité que la science s'emploie à "dévoiler ", selon l'expression consacrée.

À l'opposé, le point de vue antiréaliste défendu par van Fraassen considère que seule la partie observable du modèle scientifique se retrouve dans le monde. Plus précisément, une théorie scientifique est dite acceptable lorsque toutes les structures effectivement observées sont isomorphes aux modèles observationnels de la théorie. Un tel point de vue est en accord avec la thèse de la sous-détermination empirique des théories. Deux théories différentes affirmant l'existence d'entités théoriques différentes peuvent être équivalentes sur le plan empirique, c'est-à-dire avoir les mêmes modèles observationnels et donc être toutes deux empiriquement adéquates. Eu égard au lot d'abstractions, d'idéalisations et d'approximations qui sont monnaie commune en science, on peut donc restreindre le réalisme 
scientifique au domaine de l'observable. Un antiréalisme à part entière consisterait à dire que nous nous faisons des modèles de la réalité et ne faisons que cela. La mesure de réalité qu’il incombe d'accorder à nos meilleurs modèles ne tient pas de leur substrat ou de leur force explicative, mais de leur mode de construction.

Si la notion d'isomorphisme peut être d'un grand secours à la conception des théories scientifiques en tant que structures conceptuelles, elle n'est cependant pas à même d'apporter une solution au problème de la représentation. La relation d'isomorphisme est en effet une relation symétrique, de sorte que si $M_{1}$ est un modèle isomorphe à $M_{2}, M_{2}$ est isomorphe à $M_{1}$. À qui affirme qu'une théorie scientifique "représente » la réalité au sens d'une représentation isomorphe, on peut répliquer que la réalité est autant un modèle de la théorie que la théorie est un modèle de la réalité. Mieux vaut conserver l'ambiguïté du terme "représenter ", qui est liée à la double fonction réalisante et déréalisante de la modélisation : les modèles donnent un contenu matériel à ce qui est purement formel, et ne conservent de ce qui est matériel que ce qui est purement formel.

Pour qui adopte la conception langagière des théories scientifiques et utilise la notion de modèle au sens de la théorie des modèles, la question du réalisme scientifique se pose de la façon suivante ${ }^{11}$.

Deux résultats métalogiques sont ici d'une importance particulière. En premier lieu, il est acquis que tout système axiomatique formel du premier ordre ne détermine pas une seule structure comme domaine de validité, mais un ensemble de telles structures en tant que modèles. L'expression technique veut que de tels systèmes déterminent leur modèle visé « à isomorphisme près ». Ce défaut d'univocité dans la caractérisation axiomatique d'une structure n'a pas de quoi surprendre sur le plan intuitif. Une classe d'axiomes définissants ne peuvent discerner entre des structures identiques, même si ces structures sont composées

11 Hilary Putnam, "Models and Reality ", The Journal of Symbolic Logic, vol. 45, 1980, p. 464-482, élabore sur ces questions pour revendiquer un " réalisme interne ". 
d'éléments différents : le principe de l'identité des indiscernables vaut également pour les structures.

$\mathrm{Au}$ vu de cet argument, on peut encore défendre la thèse du réalisme scientifique en alléguant que parmi ces modèles (on emploie aussi le terme "réalisations ») que dépeint notre théorie, se trouve la réalité, le monde réel.

Cependant, un second argument d'ordre logique pose des problèmes rédhibitoires à la thèse réaliste. On dit des théories dont tous les modèles sont isomorphes qu'ils sont catégoriques. Un théorème bien connu, celui de Löwenheim-Skolem, établit que toute théorie du premier ordre contenant l'arithmétique élémentaire possède des modèles qui ne sont pas isomorphes au modèle visé. La thèse réaliste doit définitivement battre en retraite : ce défaut de catégoricité des formalismes mathématiques souligne non seulement qu'une théorie ne peut engendrer sa propre interprétation; elle indique également que cette théorie ne peut prétendre cerner la structure d'une réalité concrète visée, car il y a d'autres formes de la réalité dont la théorie est également vraie.

Il en ressort en conclusion que l'utilisation faite par la philosophie des sciences de la notion de modèle, que ce soit en tant que domaine de validité d'un ensemble d'énoncés ou en tant que structure conceptuelle directement descriptible, permet de reformuler des débats traditionnels, tel celui du réalisme scientifique, dans la lettre même - et non l'esprit - des scientifiques. 


\section{Bibliographie}

Balzer, Wolfgang, Carlos Ulises Moulines et Joseph D. Sneed, An Architectonic for Science: the Structuralist Program, Dordrecht, Kluwer, 1967.

Carnap, Rudolf, "The Methodological Character of Theoretical Concepts ", dans Feigl, Herbert et Michael Scriven (dir.), The Foundations of Science and the Concepts of Psychology and Psychoanalysis, Minneapolis, University of Minnesota Press, coll. "Minnesota Studies in the Philosophy of Science », vol. 1, 1956, p. 38-76.

Chakravartty, Anjan, "The Semantic or Model-Theoretic View of Theories and Scientific Realism ", Synthese, vol. 127, 2001, p. 325-345.

Da Costa, Newton C. A. et Steven French, "The Model Theoretic Approach in Philosophy of Science ", Philosophy of Science, vol. 57, 1990, p. 248-265.

Da Costa, Newton C. A. et Steven French, "Models, Theories, and Structures: Thirty Years on ", Philosophy of Science, vol. 67, Supplement. Proceedings of the 1998 Biennial PSA Meetings, Part II, 2000, p. 116127.

Diederich, Werner, "Realität und Modell. "Semantischer" und "strukturalistischer" Ansatz in der Wissenschaftstheorie ", Allgemeine Zeitschrift für Philosophie, vol. 14, 1989, p. 1-14.

French, Steven et James Ladyman, "Reinflating the Semantic Approach ", International Studies in the Philosophy of Science, vol. 13, 1999, p. 103121.

Giere, Ronald, Explaining Science. A Cognitive Approach, Chicago, The University of Chicago Press. 1990.

Hempel, Carl, «Studies in the Logic of Explanation », Philosophy of Science, vol. 15, 1948, p. 135-75.

Hempel, Carl, "The Theoretician's Dilemma. A Study in the Logic of Theory Construction ", dans Feigl, Herbert, Michael Scriven et Grover Maxwell (dir.), Concepts, Theories, and the Mind-Body Problem, Minneapolis, University of Minnesota Press, coll. « Minnesota Studies in the Philosophy of Science », vol. 2, 1958, p. 37-98.

Hempel, Carl, Aspects of Scientific Explanation and Other Logical Essays, New York, Free Press, 1965..

Leroux, Jean, "The Structuralist View of Theories and Empiricism ", Dialectica, vol. 41, 1987, p. 321-326.

Leroux, Jean, Une Histoire comparée de la philosophie des sciences, vol. 2, L'Empirisme logique en débat, Québec, Presses de l'Université Laval, 
coll. "Logique de la science », 2010.

Nagel, Ernest, The Structure of Science: Problems in the Logic of Scientific Explanation, New York, Hartcourt, Brace \& World, 1961.

Przełęcki, Marian, The Logic of Empirical Theories, Londres, Routledge \& Kegan Paul, 1969..

Putnam, Hilary, "Models and Reality ", The Journal of Symbolic Logic, vol. 45, 1980, p. 464-482.

Salmon, Wesley, Causality and Explanation, Oxford, Oxford University Press, 1998.

Sneed, Joseph D., The Logical Structure of Mathematical Physics, Dordrecht, Reidel, 1971.

Stegmüller, Wolfgang, The Structuralist View of Theories, Berlin, Springer, 1979.

Suppe, Frederick, The Semantic Conception of Theories and Scientific Realism, Urbana, University of Illinois Press, 1989.

Suppes, Patrick, "A Comparison of the Meaning and Use of Models in Mathematics and the Empirical Sciences ", dans Freudenthal, Hans (dir.), The Concept and the Role of Model in Mathematics and Natural and Social Science, Dordrecht, Reidel, 1961, p. 287-301.

Suppes, Patrick, "What is a Scientific Theory? ", dans Morgenbesser, Sidney (dir.), Philosophy of Science Today, New York, Basic Books, 1967, p. 55-67.

Suppes, Patrick, Newton C. A. Da Costa et Steven French, "The ModelTheoretic Approach in the Philosophy of Science ", Philosophy of Science, vol. 57, 1990, p. 248-265.

van Fraassen, Bas C., The Scientific Image, Oxford, Clarendon Press, 1980. van Fraassen, Bas C., "Empiricism in the Philosophy of Science ", dans Paul Churchland et Clifford A. Hooker (dir.), Images of Science: Essays on Realism and Empiricism with a Reply by Bas C. van Fraassen, Chicago, The University of Chicago Press, 1985, p. 245-308.

van Fraassen, Bas C., "The Semantic Approach to Scientific Theories ", dans Nancy J. Nersessian (dir.), The Process of Science, Dordrecht, Kluwer, 1987, p. 105-124.

van Fraassen, Bas C., Scientific Representation: Paradoxes of Perspective, Oxford, Clarendon Press, 2008. 